\title{
SIMULTANEOUS SUPER-RESOLUTION AND SEGMENTATION USING A GENERATIVE ADVERSARIAL NETWORK: APPLICATION TO NEONATAL BRAIN MRI
}

\author{
C.-H. Pham ${ }^{1}$, C. Tor-Díez ${ }^{1}$, H. Meunier ${ }^{2}$, N. Bednarek ${ }^{2,3}$, R. Fablet ${ }^{4}$, N. Passat ${ }^{3}$, F. Rousseau ${ }^{1}$ \\ ${ }^{1}$ IMT Atlantique, LaTIM U1101 INSERM, UBL, Brest, France \\ ${ }^{2}$ Service de médecine néonatale et réanimation pédiatrique, CHU de Reims, France \\ ${ }^{3}$ Université de Reims Champagne-Ardenne, CReSTIC, Reims, France \\ ${ }^{4}$ IMT Atlantique, Lab-STICC UMR CNRS 6285, Brest, France
}

\begin{abstract}
The analysis of clinical neonatal brain MRI remains challenging due to low anisotropic resolution of the data. In most pipelines, images are first re-sampled using interpolation or single image super-resolution techniques and then segmented using (semi-)automated approaches. Image reconstruction and segmentation are then performed separately. In this paper, we propose an end-to-end generative adversarial network for simultaneous high-resolution reconstruction and segmentation of brain MRI data. This joint approach is first assessed on the simulated low-resolution images of the high-resolution neonatal dHCP dataset. Then, the learned model is used to enhance and segment real clinical low-resolution images. Results demonstrate the potential of our proposed method with respect to practical medical applications.
\end{abstract}

Index Terms - Super-resolution, segmentation, 3D generative adversarial networks, neonatal brain MRI.

\section{INTRODUCTION}

Long-term studies of the outcome of prematurely born infants have clearly documented that the majority of such infants may have significant motor, cognitive, and behavioral deficits. However, there is a limited understanding of the nature of the cerebral abnormality underlying these adverse neurologic outcomes. Magnetic Resonance Imaging (MRI) provides unique opportunities for in vivo investigation of the early developing human brain. However, the analysis of clinical neonatal brain MRI data remains challenging mainly due to low anisotropic image resolution. Improving morphological data processing such as image resolution enhancement

\footnotetext{
The research leading to these results has been supported by the ANR MAIA project, grant ANR-15-CE23-0009 of the French National Research Agency (http://recherche.imt-atlantique.fr/maia); INSERM and Institut Mines Télécom Atlantique (Chaire "Imagerie médicale en thérapie interventionnelle"); the Fondation pour la Recherche Médicale (grant DIC20161236453); and the American Memorial Hospital Foundation. We also gratefully acknowledge the support of NVIDIA Corporation with the donation of the Titan Xp GPU used for this research.
}

and brain segmentation, is a key-point to provide robust morphometry analysis tools to the community.

One of the first key components of the processing pipeline of clinical MRI data is the upsampling image estimation. Super-resolution (SR) is a post-processing technique that aims at enhancing the resolution of an imaging system [1]. $\mathrm{SR}$ is a challenging inverse problem; in particular the estimation of texture and details remains difficult. Recently, supervised deep learning-based techniques have shown great improvement over model-based approaches. In this context, applying 3D convolutional neural networks (CNNs) yields promising results for MRI data [2,3]. However, the use of $\ell_{2}$-norm loss leads to smooth, unrealistic high resolution images $[4,5]$. Generative adversarial networks (GANs) have thus been proposed to estimate textured and sharper images $[5,6]$.

Once the high resolution image reconstruction is performed, the implementation of an automatic segmentation robust approach is crucial for fine brain structure analysis [7]. Segmenting thin structures such as the neonatal cortical gray matter remains difficult and the segmentation step is always considered separately from image reconstruction.

In this article, we propose an end-to-end GAN-based approach which can generate both the perceptually superresolved image and a cortical segmentation map from a single low-resolution (LR) image. The proposed approach called SegSRGAN is both assessed on simulated data and real clinical data.

\section{METHOD}

In this work, we focus on joint reconstruction and cortical segmentation of clinical LR neonatal brain MRI data. Section 2.1 describes the mathematical formulation of SR and Section 2.2 focuses on image segmentation. The proposed joint method is presented in Section 2.3, while technical details on the induced network architecture are provided in Section 2.4. Experiments on simulated and clinical MRI data are described and discussed in Section 3. 


\subsection{Formulation of single image super-resolution}

The objective of single image SR is to estimate a highresolution (HR) image $\mathbf{X} \in \mathbb{R}^{m}$ from one observed LR image $\mathbf{Y} \in \mathbb{R}^{n}$. SR problem can be formulated using the following linear observation model:

$$
\mathbf{Y}=H_{\downarrow} B \mathbf{X}+N=\Theta \mathbf{X}+N
$$

where $N$ is the additive noise, $B \in \mathbb{R}^{m \times m}$ is a blur matrix (depending on the point spread function), $H_{\downarrow}: \mathbb{R}^{m} \rightarrow \mathbb{R}^{n}$ is a downsampling decimation and $\Theta=H_{\downarrow} B \in \mathbb{R}^{n \times m}(m>n)$.

A popular approach that solves SR problem defines the matrix $\Theta^{-1}$ as the combination of a restoration operator $F \in$ $\mathbb{R}^{m \times m}$ and an upscaling interpolation operator $S^{\uparrow}: \mathbb{R}^{n} \rightarrow$ $\mathbb{R}^{m}$ computing the interpolated LR image $\mathbf{Z} \in \mathbb{R}^{m}$ ( $\mathbf{Z}=$ $S^{\uparrow} \mathbf{Y}$ ). In the context of supervised learning, given a set of HR images $\mathbf{X}_{i}$ and their corresponding LR images $\mathbf{Y}_{i}$, the restoration operator $F$ can be estimated by minimizing the following loss function: $\widehat{F}=\arg \min _{F} \sum_{i}\left\|\mathbf{X}_{i}-F\left(\mathbf{Z}_{i}\right)\right\|_{2}^{2}$.

However, it is known that the use of $\ell_{2}$-norm may lead to oversmoothing high resolution images. In order to provide realistic HR images, perceptual loss function [4] have been used in a GAN [5]. This is a paradigm shift since it is no longer a question of minimizing only the reconstruction error but of estimating a realistic image, i.e. a high resolution image that corresponds to the observation model with realistic texture details.

A perceptual loss can be formulated as the weighted sum of the content loss (based, e.g., on pixel-wise mean squared error loss) and an adversarial loss component. In GAN-based approaches, the purpose is to train a generating network $G$ that estimates for a given LR input image a corresponding HR image. The goal of the discriminator network $D$ is to classify real and simulated HR images.

\subsection{Formulation of image segmentation}

In this work, image segmentation is viewed as a supervised regression problem: $\mathbf{S}_{\mathbf{X}}=R(\mathbf{X})$ where $R$ denotes a non-linear mapping from the upscaled image $\mathbf{X}$ to the segmentation map $\mathbf{S}_{\mathbf{X}}$. Similarly to the SR problem, assuming that we have a set of images $\mathbf{X}_{i}$ and corresponding segmentation maps $\mathbf{S}_{\mathbf{X}_{i}}$, a general approach for solving this segmentation problem is to find the mapping $R$ by minimizing the following loss function: $\widehat{R}=\arg \min _{R} \sum_{i}\left\|\mathbf{S}_{\mathbf{X}_{i}}-R\left(\mathbf{X}_{i}\right)\right\|_{2}^{2}$. Unlike the SR problem, the use of $\ell_{2}$-norm is less critical as it is expected to estimate smooth segmentation maps.

\subsection{Joint mapping by generative adversarial networks}

We propose the use of a GAN-based approach to estimate jointly a HR image and its corresponding segmentation map from one LR image. To this end, a convolution-based generator network $G$ takes as input an interpolated LR image $\mathbf{Z}$ and computes a HR image $\widehat{\mathbf{X}}$ and a segmentation map $\widehat{\mathbf{S}_{\mathbf{X}}}$ by minimizing the following reconstruction loss:

$$
\mathcal{L}_{\text {rec }}=\min _{G} \sum_{i} \rho\left(\left(\mathbf{X}, \mathbf{S}_{\mathbf{X}}\right)_{i}-G\left(\mathbf{Z}_{i}\right)\right)
$$

where $\left(\mathbf{X}, \mathbf{S}_{\mathbf{X}}\right)_{i}$ are concatenated along the feature channel. In this work, we use a robust loss as Charbonnier loss [8]: $\rho(x)=\sqrt{x^{2}+\nu^{2}}$ where $\nu$ is set to $10^{-3}$.

The discriminator network $D$ attempts to distinguish the real data $\left(\mathbf{X}, \mathbf{S}_{\mathbf{X}}\right)$ and the generated ones $G(\mathbf{Z})$. The game between the generator $G$ and the discriminator $D$ is usually modeled with a minimax objective. However, using such loss function, GAN may be unstable or can suffer from mode collapse during training. Thus, in this work, we propose to use Wasserstein GAN loss described in [9]:

$$
\begin{aligned}
\mathcal{L}_{a d v} & =\min _{G} \max _{D} \mathbb{E}_{\mathbf{X} \sim \mathbb{P}_{\mathbf{X}}, \mathbf{S}_{\mathbf{X}} \sim \mathbb{P}_{\mathbf{X}}}\left[D\left(\left(\mathbf{X}, \mathbf{S}_{\mathbf{X}}\right)\right)\right]- \\
& \mathbb{E}_{\mathbf{Z} \sim \mathbb{P}_{\mathbf{Z}}}[D(G(\mathbf{Z}))]+\lambda_{g p} \mathbb{E}_{\widehat{\mathbf{X S}}}\left[\left(\|\left(\nabla_{\widehat{\mathbf{X S}}} D(\widehat{\mathbf{X S}}) \|_{2}-1\right)^{2}\right]\right.
\end{aligned}
$$

where $\widehat{\mathbf{X S}}$ is the interpolation of the true data and the generated one as $(1-\epsilon)\left(\mathbf{X}, \mathbf{S}_{\mathbf{X}}\right)+\epsilon G(\mathbf{Z}), \epsilon \sim \mathcal{U}[0,1]$ (uniform distribution). $\lambda_{g p}$ and $\nabla$ denote the gradient penalty coefficient and gradient operator, respectively. The images $\mathbf{X}, \mathbf{S}_{\mathbf{X}}$ and $\mathbf{Z}$ are extracted randomly from the data distributions of $\mathrm{HR}$ images $\mathbb{P}_{\mathbf{X}}$, HR segmentation maps $\mathbb{P}_{\mathbf{S}_{\mathbf{X}}}$ and LR images $\mathbb{P}_{\mathbf{Z}}$. The terms $D\left(\left(\mathbf{X}, \mathbf{S}_{\mathbf{X}}\right)\right), D(G(\mathbf{Z}))$ and $D(\widehat{\mathbf{X S}})$ are the responses of the discriminator with respect to the real data, the generated data and the interpolated data, respectively. The full objective function is expressed as: $\mathcal{L}_{\text {total }}=\mathcal{L}_{\text {rec }}+\lambda_{\text {adv }} \mathcal{L}_{a d v}$ where $\lambda_{a d v}$ is a trade-off parameter between reconstruction loss and adversarial loss.

\subsection{Architecture of generator and discriminator net- works}

The generator network is a convolution-based network with residual blocks. Let $C_{j}^{i}-S^{k}$ be a block consisting of the following layers: a convolution layer of $j$ filters of size $i^{3}$ with stride of $k$, an instance normalization layer (InsNorm) [10] and a rectified linear unit (ReLU). $R_{k}$ denotes a residual block as Conv-InsNorm-ReLU-Conv-InsNorm that contains $3^{3}$ convolution layers with $k$ filters. $U_{k}$ denotes layers as Upsampling-Conv-InsNorm-ReLU layer with $k$ filters of $3^{3}$ and stride of 1 . After the last layer, we apply a sigmoid activation for the channel of segmentation map and an element-wise sum of the intensity channel and the interpolated LR image. The generator architecture is: $C_{16}^{7}{ }^{-} S^{1}, C_{32}^{3}{ }^{2} S^{2}, C_{64}^{3}{ }^{-} S^{2}, R_{64}$, $R_{64}, R_{64}, R_{64}, R_{64}, R_{64}, U_{32}, U_{16}, C_{2}^{7}-S^{1}$.

The discriminator network contains five convolutional layers with an increasing number of filter kernels, increasing by a factor of 2 from 32 to 512 kernels. Let $C_{k}$ be a block consisting of the following layers: a convolution layer of $k$ filters of size $4^{3}$ with stride of 2 and a Leaky ReLU with a 
negative slope of 0.01 . The last layer $C_{1}^{2}$ is a $2^{3}$ convolution filter with stride of 1 . No activation layer is used after the last layer. The discriminator consists of $C_{32}, C_{64}, C_{128}, C_{256}$, $C_{512}, C_{1}^{2}$.

\section{EXPERIMENTS AND RESULTS}

\subsection{Datasets and network training}

To assess the ability to reconstruct HR volume and segment the cerebral cortex , we applied the proposed method on T2weighted (T2w) MR images of the developing Human Connectome Project ${ }^{1}$ (dHCP). 40 T2w images were acquired using a $3 \mathrm{~T}$ Achieva scanner with a $0.5 \times 0.5 \times 0.5 \mathrm{~mm}^{3}$ resolution with $\mathrm{TR}=12000 \mathrm{~ms} \mathrm{TE}=156 \mathrm{~ms}$, respectively. 30 images were used for training networks, whereas the other 10 were used as testing images. As in [1], LR images were generated by using a Gaussian blur with the full-width-at-halfmaximum (FWHM) set to slice thickness before a downsampling step to obtain a $0.5 \times 0.5 \times 1.5 \mathrm{~mm}^{3}$ resolution.

We have also applied the proposed method onto clinical neonatal MRI data acquired in the neonatology service of Reims Hospital. These LR images have a resolution of $0.446 \times 0.446 \times 3 \mathrm{~mm}^{3} .40 \mathrm{HR}$ images of the dataset $\mathrm{dHCP}$ were filtered and downsampled as in [1] in order to generate LR images with a same resolution as clinical data. The network was trained using 40 pairs of simulated data and then applied to real LR images for visual evaluations. All data had bias correction and for network training, they were normalized between 0 and 1 . No subjects nor image patches appear twice in the different subsets.

The 3D network was trained over 200 epochs with batch size of 16, using Adam method with learning rate of 0.0001 and updates the discriminator 5 times before training the generator as in [9]. The parameters $\lambda_{a d v}$ and $\lambda_{g p}$ were set to 0.001 and 10 respectively. The training patch size is $64^{3}$. At test time, the whole HR image and segmentation volume were reconstructed by the weighted predictions of patches. A thresholding at 0.5 has been performed to obtain binary segmentation maps.

\subsection{Results}

Peak signal-to-noise ratio (PSNR) and structural similarity index (SSIM) have been used to evaluate the performance of SR reconstructions. Table 1 provides a summary of quantitative evaluations for the following methods: cubic spline interpolation, a 20-layers CNN-based SR approach (20LSRReCNN) [11] and our proposed SegSRGAN. It can be seen that 20L-SRReCNN provides highest PSNRs as in [4, 5] since this approach minimizes a $\ell_{2}$-norm-based loss. However, while the two CNN-based approaches (20L-SRReCNN and SegSRGAN) lead qualitatively to similar realistic results

\footnotetext{
${ }^{1}$ http://www.developingconnectome.org
}

Table 1. Quantitative evaluation of SR methods on dHCP dataset.

\begin{tabular}{cccc} 
& Interpolation & 20L-SRReCNN [11] & SegSRGAN \\
\hline PSNR & 30.70 & 35.84 & 31.75 \\
\hline SSIM & 0.9492 & 0.9739 & 0.9624 \\
\hline
\end{tabular}

Table 2. Quantitative evaluation of segmentation methods on dHCP dataset.

\begin{tabular}{lccc}
\hline & IMAPA [12] & DrawEM [13] & SegSRGAN \\
\hline Dice (mean) & 0.788 & 0.818 & 0.886 \\
\hline Dice (std. dev.) & 0.061 & 0.014 & 0.011 \\
\hline
\end{tabular}

on dHCP dataset (see Fig. 1), the proposed approach provides the best reconstructed HR image on clinical data with better contrast on cortical gray matter (see Fig. 2).

The Dice index is used to evaluate the cortical segmentation maps obtained by the following state-of-the-art methods: iterative multi-atlas patch-based approach (IMAPA) [12], DrawEM [13] and the proposed SegSRGAN. As in a typical clinical setting, the three methods have been applied on interpolated images. Table 2 shows that quantitatively the proposed approach lead to the best cortical segmentation results with significant improvement with respect to the two other methods. Moreover, as mentioned in [12], the use of IMAPA applied on original HR dHCP images leads to a mean DICE of 0.887 (std. dev. of 0.011 ) that is very similar to the results obtained with SegSRGAN (applied on interpolated images). Results on real LR data (see Fig. 2) confirm the potential of the proposed approach for fine analysis of clinical neonatal brain MRI.

\section{CONCLUSION}

In this paper, we have presented a simultaneous superresolution and segmentation method for 3D brain MR images using a generative adversarial network. Our experiments on both simulated and clinical data have shown that better performance can be achieved by this joint approach compared to state-of-the-art techniques, opening up new perspectives in the processing of clinical LR neonatal brain MRI data.

\section{REFERENCES}

[1] H. Greenspan, "Super-resolution in medical imaging," The Computer Journal, vol. 52, no. 1, pp. 43-63, 2008.

[2] C.-H. Pham, A. Ducournau, R. Fablet, and F. Rousseau, "Brain MRI super-resolution using deep 3D convolutional networks," in IEEE ISBI, 2017, pp. 197-200.

[3] Y. Chen, Y. Xie, Z. Zhou, F. Shi, A. G. Christodoulou, and D. Li, "Brain MRI super resolution using 3D deep densely connected neural networks," in IEEE ISBI, 2018, pp. 739-742. 


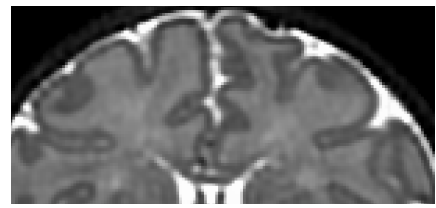

(a) Original $\mathrm{dHCP} \mathrm{HR}$

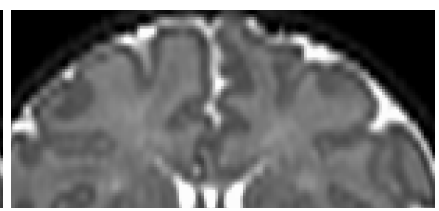

(b) Spline interpolation

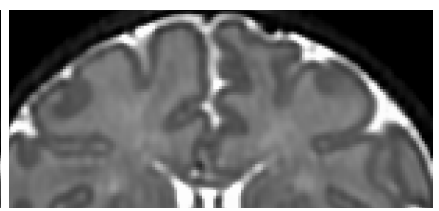

(c) 20L-SRReCNN [11]

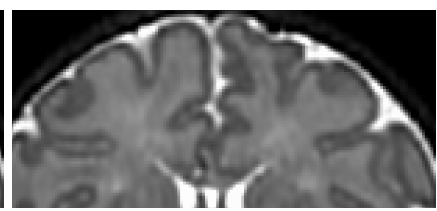

(d) Proposed approach

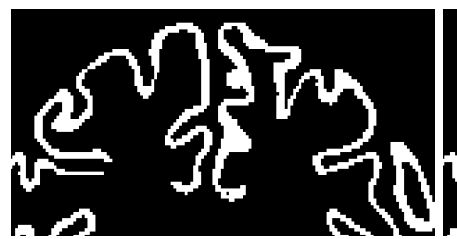

(e) Original dHCP segmentation

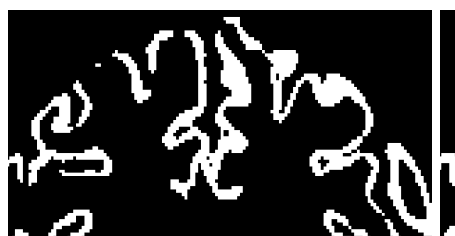

(f) IMAPA [12]

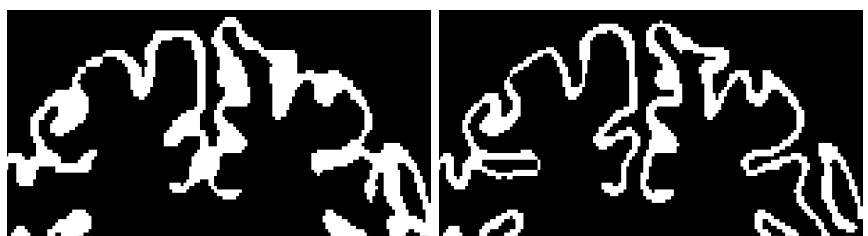

(g) DrawEM [13]

(h) Proposed approach

Fig. 1. SR and segmentation results for one dHCP subject: (a) original HR image; (b-d) SR reconstruction of the LR image generated from (a); (e) segmentation ground-truth of (a); (f,g) segmentation of (b); (h) HR segmentation from the LR image using the joint SegSR-GAN method.

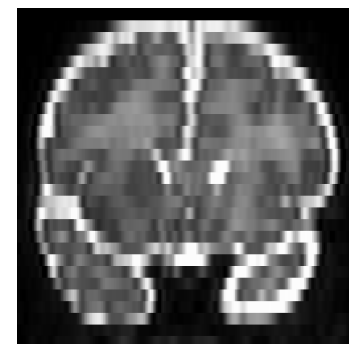

(a) LR original image

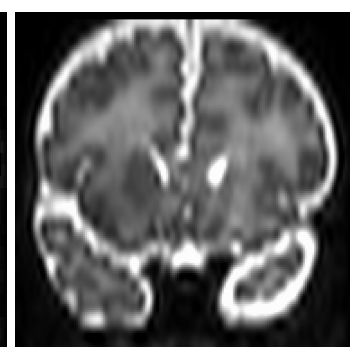

(b) Spline interpolation

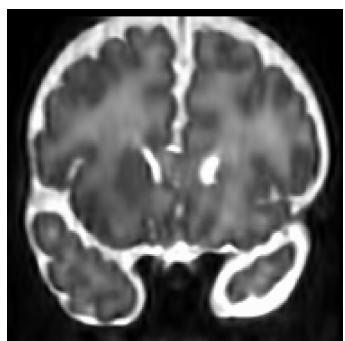

(c) 20L-SRReCNN [11]

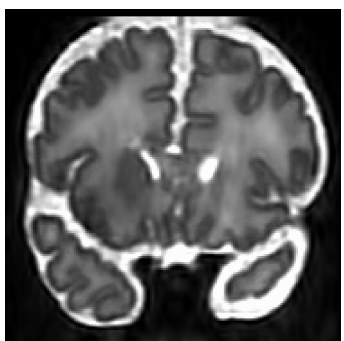

(d) Proposed approach

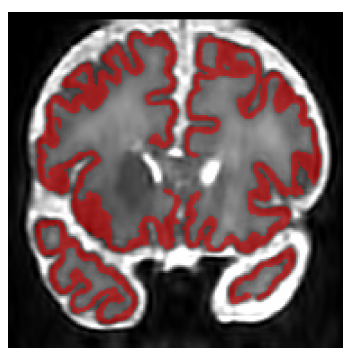

(e) Proposed approach

Fig. 2. Reconstruction (b-d) and segmentation results (e) on a real LR neonatal brain image (a) with voxel size of $0.446 \times$ $0.446 \times 3 \mathrm{~mm}^{3}$, re-sampled to $0.5 \times 0.5 \times 0.5 \mathrm{~mm}^{3}$.

[4] J. Johnson, A. Alahi, and L. Fei-Fei, "Perceptual losses for real-time style transfer and super-resolution," in ECCV, 2016, pp. 694-711.

[5] C. Ledig et al., "Photo-realistic single image superresolution using a generative adversarial network," in IEEE CVPR, 2017, pp. 105-114.

[6] Y. Chen, F. Shi, A. G. Christodoulou, Y. Xie, Z. Zhou, and D. Li, "Efficient and accurate MRI super-resolution using a generative adversarial network and 3D multilevel densely connected network," in MICCAI, 2018, pp. 91-99.

[7] A. Makropoulos, S. J. Counsell, and D. Rueckert, "A review on automatic fetal and neonatal brain MRI segmentation," NeuroImage, vol. 170, pp. 231-248, 2017.

[8] P. Charbonnier, L. Blanc-Féraud, G. Aubert, and M. Barlaud, "Deterministic edge-preserving regularization in computed imaging," IEEE Transactions on Image Processing, vol. 6, no. 2, pp. 298-311, 1997.
[9] I. Gulrajani, F. Ahmed, M. Arjovsky, V. Dumoulin, and A. C. Courville, "Improved training of Wasserstein GANs," in NIPS, 2017, pp. 5769-5779.

[10] D. Ulyanov, A. Vedaldi, and V. S. Lempitsky, "Improved texture networks: Maximizing quality and diversity in feed-forward stylization and texture synthesis," in IEEE CVPR, 2017, pp. 4105-4113.

[11] C.-H. Pham, R. Fablet, and F. Rousseau, "Multi-scale brain MRI super-resolution using deep 3D convolutional networks," Preprint hal-01635455, 2017.

[12] C. Tor-Díez, N. Passat, I. Bloch, S. Faisan, N. Bednarek, and F. Rousseau, "An iterative multi-atlas patch-based approach for cortex segmentation from neonatal MRI," Computerized Medical Imaging and Graphics, vol. 70, pp. 73-82, 2018.

[13] A. Makropoulos et al., "Automatic whole brain MRI segmentation of the developing neonatal brain," IEEE Transactions on Medical Imaging, vol. 33, no. 9, pp. 1818-1831, 2014. 